A. Schulte-Mecklenbeck

Klinik für Neurologie mit Institut für Translationale Neurologie, Universitätsklinikum Münster, Münster, Deutschland

\title{
Entzündungsmediatoren im Liquor verbessern die Prognose bei Multipler Sklerose
}

\section{Originalpublikation}

Magliozzi R, Scalfari A, Pisani Al et al. (2020) The CSF profile linked to cortical damage predicts multiple sclerosis activity. Ann Neurol 88:562-573. https://doi.org/10.1002/ana.25786

Bisher gibt es wenige Anhaltspunkte, anhand derer sich der Krankheitsverlauf von Patienten mit schubförmiger multipler Sklerose prognostizieren lässt. Dies ist jedoch eine Voraussetzung dafür, möglichst frühzeitig eine Immuntherapie mit ausreichender Wirkstärke zu initiieren und so den Krankheitsverlauf nachhaltig zu beeinflussen.

Zurzeit werden Entzündungsparameter im Liquor hauptsächlich zur Diagnosestellung sowie Differenzialdiagnostik verwendet. In der multiplen Sklerose geht intrathekale Entzündung mit neuronalem Zelluntergang in der grauen Substanz einher, der wiederum stark mit dem langfristigen Grad der Behinderung korreliert [1-4]. Daher untersuchten die Autoren dieser Studie das prognostische Potenzial einer Auswahl von intrathekalen Entzündungsmediatoren für den weiteren Krankheitsverlauf. Zu diesem Zweck wurde der Liquor von 99 neu diagnostizierten MS-Patienten per Luminex-Technologie untersucht. Die Ergebnisse wurden u. a. mit der Entstehung neuer kortikaler Läsionen und der Krankheitsaktivität im weiteren Verlauf von 4 Jahren verglichen. Dabei wurde Krankheitsaktivität als das Auftreten von Schüben, neuen T2-Läsionen in der weißen Substanz oder einer Erhöhung in der „expanded disability status scale" (EDSS) um 1 Punkt definiert. Die eingeschlossenen Patienten erhielten eine immunmodulatorische Basistherapie mit Interferon beta-1a, Glatiramerazetat, Teriflunomid oder Dimethylfumarat.

Die Autoren konnten zeigen, dass Patienten mit fortschreitender Krankheitsaktivität unter Basistherapie einen signifikant erhöhten Anteil von CXCL13, CXCL12, INF- $\gamma$, TNF, sCD163, LIGHT und APRIL an den gesamten intrathekalen Proteinen haben. Gleichzeitig zeigte sich, dass CXCL13, TNF, sCD163, LIGHT und TWEAK mit einer Verdünnung des Kortex einhergehen. Durch die Integration der beschriebenen intrathekalen Entzündungsparameter ließ sich die Prognosegüte für ein Fortschreiten der Krankheitsaktivität unter Basistherapie im Vergleich zur Prognose nur aufgrund von klinischen und
MRT-Daten von $44 \%$ auf $71 \%$ für den Zeitraum von 4 Jahren erhöhen. Darüber hinaus deutet die Assoziation der meisten identifizierten Parameter mit der Rekrutierung von B-Zellen, T-Zell-Aktivität, Mikrogliazellen und der Entstehung von tertiären lymphatischen Strukturen einmal mehr auf die komplexen Immunpathogene der MS hin.

\section{Kommentar}

Diese Studie liefert einen weiteren wichtigen Baustein für die klinische Nutzung intrathekaler Entzündungsparameter wie CXCL13, INF- $\gamma$, TNF für eine zukünftige patientenindividuelle Therapie der schubförmigen multiplen Sklerose. Um diese Erkenntnisse für die Mehrzahl der Patienten nutzbar zu machen, sollten als Nächstes robuste Grenzwerte für die Einteilung neu diagnostizierter Patienten in Risikogruppen erarbeitet und validiert werden.
Literatur

1. Kutzelnigg A, Lucchinetti CF, Stadelmann Cet al (2005) Cortical demyelination and diffuse white matter injury in multiple sclerosis. Brain 128:2705-2712

2. Magliozzi R, Howell OW, Reeves C et al (2010) A gradient of neuronal loss and meningeal inflammation in multiple sclerosis. Ann Neurol 68:477-493

3. Calabrese M, Magliozzi R, Ciccarelli O et al (2015) Exploring the origins of grey matter damage in multiple sclerosis. Nat Rev Neurosci 16:147-158

4. Scalfari A, Romualdi C, Nicholas RS et al (2018) The cortical damage, early relapses, and onset of the progressive phase in multiple sclerosis. Neurology 90:e2107-e2118

\section{Korrespondenzadresse}

\section{Dr. rer. nat. A. Schulte-Mecklenbeck}

Klinik für Neurologie mit Institut für Translationale

Neurologie

Universitätsklinikum Münster

Albert-Schweitzer Campus 1A1, 48149 Münster,

Deutschland

andreas.schulte-mecklenbeck@ukmuenster.de

\section{Redaktion}

L. Klotz, Münster

DGNeurologie $2021 \cdot 4$ (1): 58

https://doi.org/10.1007/s42451-020-00272-9

Online publiziert: 9. Dezember 2020

(c) Springer Medizin Verlag GmbH, ein Teil von Springer Nature 2020 